\title{
Multiple diffraction lines in the synchrotron x-ray topographs of elastically bent silicon single crystals
}

\author{
T. Tuomi and K. Naukkarinen \\ Laboratory of Physics, Helsinki University of Technology, SF-02150 Espoo 15, Finland
}

(Received 21 May 1981)

\begin{abstract}
X-ray topographs of elastically bent silicon crystals taken with continuous spectrum of a parallel synchrotron radiation beam show straight and curved intersecting lines. These lines are interpreted as due to simultaneous reflection by at least two lattice planes. The interpretation is supported by detailed calculations carried out for a double diffraction case $(4 \overline{4} 2,3 \overline{3} 1 ; 1 \overline{1} 1)$, in which one reflection $(4 \overline{4} 2)$ is forbidden in single diffraction.
\end{abstract}

$\mathrm{X}$-ray diffraction topographs of a silicon single crystal taken with synchrotron radiation which has a continuous spectrum show weak (white) and strong (black) intensity lines if the sample is elastically bent. ${ }^{1}$ For the same reason, such lines appear in the synchrotron $\mathrm{x}$-ray topographs of epitaxially grown semiconductor laser crystals ${ }^{2}$ and in those of strained iron polycrystals. ${ }^{3}$ A systematic study was undertaken with a dislocation-free silicon single crystal in order to understand the physical phenomenon leading to the appearance of these lines not previously reported elsewhere.

The silicon sample was a rectangular $0.36-\mathrm{mm}$ thick (001) plate measuring $17 \times 36 \mathrm{~mm}^{2}$. Its one end was fixed and the other end was pushed with a plastic finger so that the sample was bent about a [110] vertical axis. In the unstrained state the crystal (001) face was nearly perpendicular to the incident synchrotron radiation beam, the diameter of which was $6 \mathrm{~mm}$. Laue transmission sets of circular diffracted beam spots were taken on Kodak $\mathrm{R}$ single-coated films placed $45 \mathrm{~mm}$ behind the crystal perpendicularly to the synchrotron radiation beam. Relative strain was measured with a strain gauge glued on the surface of the crystals.

At small values of bending (radius of curvature of the sample from $2-4 \mathrm{~m})$ rather broad $(\sim 1$ $\mathrm{mm}$ ) stripes were observed in a few spots. The black and white contrast of these fringes is faint and it is possible that in most spots they merge into the increased background intensity. The fringes are reminiscent of the Pendellösung fringes, ${ }^{4}$ which have also been observed with polychromatic synchrotron radiation in the transmission topographs of a wedge-shaped silicon crystal. ${ }^{5,6}$ They can be interpreted according to the dynamical theory of $\mathrm{x}$-ray diffraction as equal-inclination fringes.
When the sample was bent further so that its radius of curvature became smaller than $2 \mathrm{~m}$, distinct white (small $x$-ray intensity) and less frequently black (large intensity) lines nearly parallel to the bending axis appeared in several Laue spots such as those shown in Figs. 1(a) and 1(b). The two topographs of Figs. 1(a) and 1(b) are $4 \overline{4} 2$ and $3 \overline{3} 1$ reflections, respectively. One of them, $4 \overline{4} 2$, is forbidden, i.e., its structure factor $F_{2}=0$, the other, $3 \overline{3} 1$, is a rather strong reflection: $F_{1}=4 \sqrt{2} f$, where $f$ is the atomic scattering factor of silicon. In this case $f=40$. It is evident from this observation that a rather narrow nearly vertical part of the crystal is turned into a position in which $4 \overline{4} 2$ is made allowed through an Umweg. The situation is depicted in the reciprocal lattice in Fig. 1(c). The wave vector $\overrightarrow{\mathrm{k}}_{2}$ of the reflection $4 \overline{4} 2$ is the sum of the vectors $\overrightarrow{\mathrm{k}}_{1}$ (reflection $3 \overline{3} 1$ ) and $\overrightarrow{\mathrm{H}}_{2}-\overrightarrow{\mathrm{H}}_{1}$, which is equal to a reciprocal-lattice vector,from the origin to the point $1 \overline{1} 1$. This multiple diffraction is called three-beam case or double diffraction, and it is written $(4 \overline{4} 2,3 \overline{3} 1,1 \overline{1} 1)$ according to the equation

$$
\overrightarrow{\mathrm{k}}_{2}-\overrightarrow{\mathrm{k}}_{1}=\overrightarrow{\mathrm{H}}_{2}-\overrightarrow{\mathrm{H}}_{1}
$$

obtained by subtraction from two Bragg equations

$$
\begin{aligned}
& \overrightarrow{\mathrm{k}}_{2}-\overrightarrow{\mathrm{k}}_{0}=\overrightarrow{\mathrm{H}}_{2}, \\
& \overrightarrow{\mathrm{k}}_{1}-\overrightarrow{\mathrm{k}}_{0}=\overrightarrow{\mathrm{H}}_{1},
\end{aligned}
$$

in which $\overrightarrow{\mathrm{k}}_{0}$ is the wave vector of the incoming beam and $\overrightarrow{\mathrm{H}}_{1}$ and $\overrightarrow{\mathrm{H}}_{2}$ are the reciprocal-lattice vectors $[3 \overline{3} 1]$ and $[4 \overline{4} 2]$, respectively.

The intensities of the multiply diffracted beams can be estimated in a kinematical approximation as done by Moon and Shull ${ }^{7}$ for neutrons and $\mathrm{Za}$ chariasen ${ }^{8}$ for $\mathrm{x}$ rays. Using a beam of monochromatic $\mathrm{x}$ rays an increase of intensity of a forbidden reflection Umweganregung was observed by 

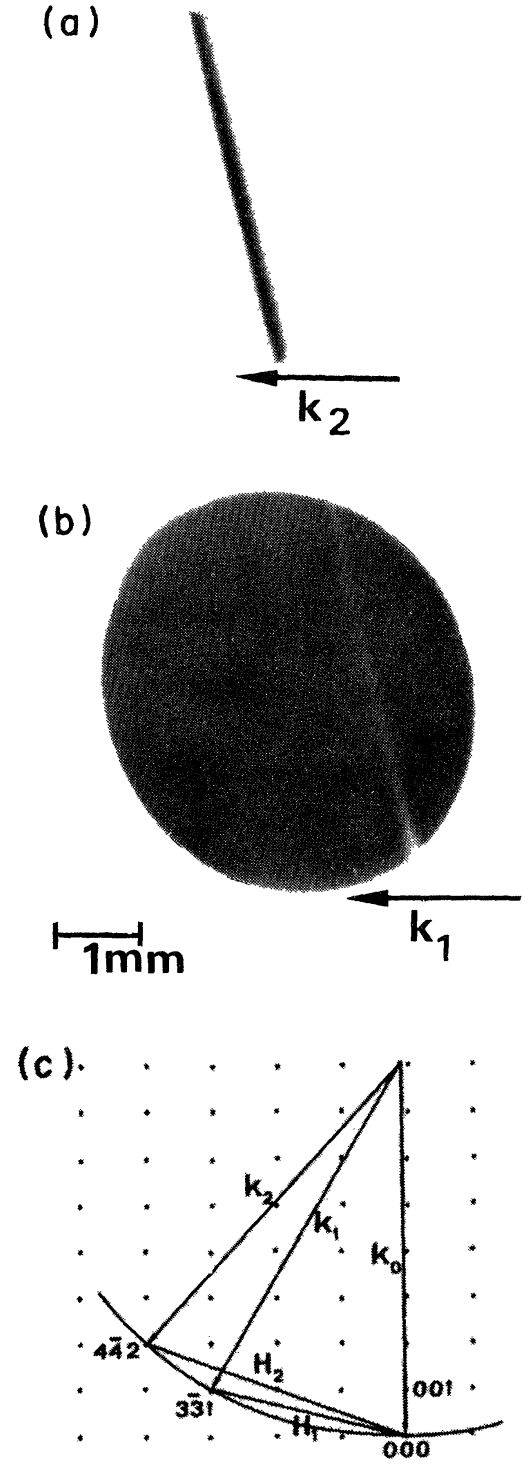

FIG. 1. (a) $4 \overline{4} 2$ and (b) $3 \overline{3} 1$ transmission topographs of a (001) $0.36-\mathrm{mm}$ thick silicon single-crystal plate bent about the vertical [110] axis showing a line at which the double diffraction condition depicted in the reciprocal lattice (c) is fulfilled. The reflection $4 \overline{4} 2$ is forbidden in single diffraction. The exposure time on Kodak $\mathbf{R}$ film was $1 \mathrm{~s}$. The DORIS synchrotron radiation source parameters were $3.3 \mathrm{GeV}$ and $40 \mathrm{~mA}$. The measured strain on the surface of the sample was $3.5 \times 10^{-4}$.

Renninger in an unstrained crystal ${ }^{9}$ and by Borrmann with a diverging beam in anomalous transmission. ${ }^{10}$ The opposite phenomenon, a decrease of intensity of a strong reflection called Aufhellung, has been reported first by Wagner. ${ }^{11}$ In this work a parallel beam having a continuous (a)

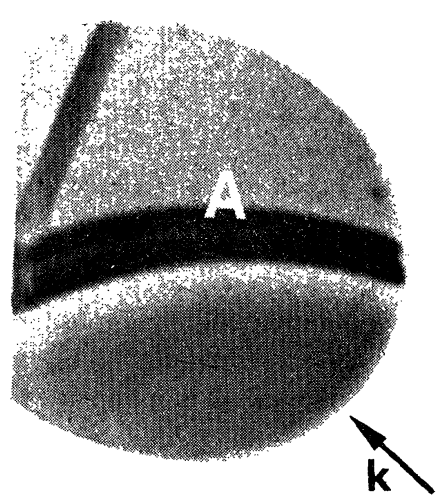

(b)

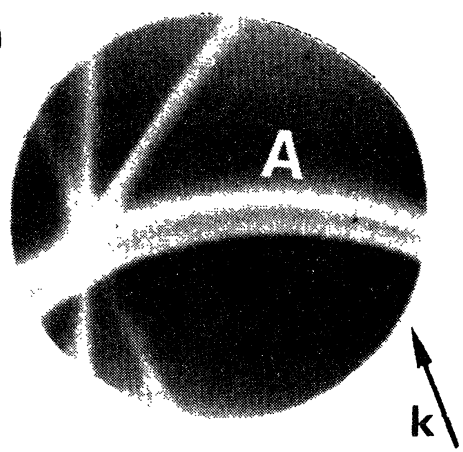

(c)

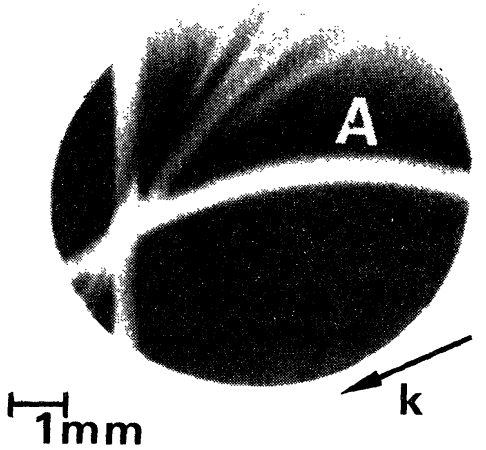

FIG. 2. (a) $1 \overline{5} 3$, (b) $2 \overline{4} 2$, and (c) $3 \overline{1} 1$ transmission topographs of a bent silicon sample showing a triple diffraction line $A$.

spectrum is used and multiple diffraction is made possible by bending the crystal.

In the case of Fig. 1(c) let the intensities in the directions of $\overrightarrow{\mathrm{k}}_{0}, \overrightarrow{\mathrm{k}}_{1}$, and $\overrightarrow{\mathrm{k}}_{2}$ be $P_{0}, P_{1}$, and $P_{2}$, respectively. Intensity losses due to diffraction by the lattice planes $(3 \overline{3} 1),(4 \overline{4} 2)$, and (1 $1 \overline{1} 1)$ are described by the reflecting powers $\sigma_{1}, \sigma_{2}$, and $\sigma_{3}$, respectively. Absorption is assumed negligible. At depth $x$ below the entrance surface of the crystal the following equations can be written for the derivatives of the intensities: 


$$
\begin{aligned}
& \frac{d P_{0}}{d x}=-\left(\sigma_{1}+\sigma_{2}\right) P_{0}+\frac{1}{\gamma_{1}} \sigma_{1} P_{1}+\frac{1}{\gamma_{2}} \sigma_{2} P_{2}, \\
& \frac{d P_{1}}{d x}=\sigma_{1} P_{0}-\frac{1}{\gamma_{1}}\left(\sigma_{1}+\sigma_{3}\right) P_{1}+\frac{1}{\gamma_{2}} \sigma_{3} P_{2}, \\
& \frac{d P_{2}}{d x}=\sigma_{2} P_{0}+\frac{1}{\gamma_{1}} \sigma_{3} P_{1}-\frac{1}{\gamma_{2}}\left(\sigma_{2}+\sigma_{3}\right) P_{2},
\end{aligned}
$$

where $\gamma_{1}$ and $\gamma_{2}$ are the direction cosines of the beams $\overrightarrow{\mathrm{k}}_{1}$ and $\overrightarrow{\mathrm{k}}_{2}$, respectively, relative to the incident beam $\overrightarrow{\mathrm{k}}_{0}$. The reflecting powers are

$$
\sigma_{i}=W(\Delta)\left|e^{2} F_{i} /\left(4 \pi \epsilon_{0} m c^{2} V\right)\right|^{2} \lambda^{3} \cos ^{2} 2 \theta_{B},
$$

where $W(\Delta)$ is the distribution function describing the misalignment of mosaic blocks. Although the crystal is homogenously bent, it is assumed that it can be replaced for the mathematical treatment by submicroscopic crystallites and that the half-width of $W(\Delta)$ is much larger than that of a perfect single block diffraction curve. $\Delta$ is a small angle measured from the center of the double diffraction angular position. Using the measured value of the Bragg angle $\theta_{B}$, the structure factors $F_{i}$ are calculated. Inserting the values of $F_{i}$ into Eq. (5) one obtains $\sigma_{2}=0$ and $\sigma_{3} \approx 2 \sigma_{1}$. If $S_{0}$ is the incident intensity at the wavelength $\lambda=64 \mathrm{pm}$ of the double diffraction the boundary conditions on the entrance face $x=0$ are $P_{0}(0)=S_{0}, P_{1}(0)=0$, and $P_{2}(0)=0$. Taking $\gamma_{1}=\gamma_{2}=1$ the solution of Eq. (4) is

$$
\begin{gathered}
P_{0}=\left(S_{0} / 3\right)\left[1+\frac{1}{2}(2+\sqrt{3}) \exp \left(r_{2} x\right)\right. \\
\left.+\frac{1}{2}(2-\sqrt{3}) \exp \left(r_{3} x\right)\right], \\
P_{1}=\left(S_{0} / 3\right)\left[1-\frac{1}{2} \exp \left(r_{2} x\right)-\frac{1}{2} \exp \left(r_{3} x\right)\right], \\
P_{2}=\left(S_{0} / 3\right)\left[1-\frac{1}{2}(\sqrt{3}+1) \exp \left(r_{2} x\right)\right. \\
\left.+\frac{1}{2}(\sqrt{3}-1) \exp \left(r_{3} x\right)\right]
\end{gathered}
$$

with $r_{2}=(\sqrt{3}-3) \sigma_{1}$ and $r_{3}=-(\sqrt{3}+3) \sigma_{1}$. The observable intensities $\bar{P}_{i}=\int P_{i}(\Delta) d \Delta$ are calculated from Eq. (6) using series expansions of the exponential functions. Taking only the first few terms of the series $P_{i}$ can be written as in Table I, in which also the intensities for the single diffraction case are given for comparison.

The values of $\bar{P}_{i 2}$ (double diffraction) and $\bar{P}_{i 1}$ (single diffraction) were measured with a microdensitometer from the original films of Figs. 1(a) and 1(b). From them experimental values $\bar{P}_{12} / \bar{P}_{11}$ $=0.72$ and $\bar{P}_{22} / \bar{P}_{11}=0.35$ were obtained. In order to calculate these intensity ratios $W(\Delta)$ is first estimated from the width of the double diffraction line in Figs. 1(a) and 1(b). The 0.15-mm-wide line covers the angular range of $0.24 \mathrm{mrad}$ of the lattice planes, because in the experiment of Fig. 1 the relative strain on the surface of the sample was $3.5 \times 10^{-4}$, corresponding to a radius of curvature of $0.5 \mathrm{~m}$. Taking $W(\Delta)=4200$ in the range -0.12 $\operatorname{mrad} \leq \Delta \leq 0.12 \mathrm{mrad}$ and $W(\Delta)=0$ elsewhere, $g=4200$ is obtained. Inserting now $g=4200$, $Q_{1}=0.13 \mathrm{~m}^{-1}$, and $x_{0}=0.36 \mathrm{~mm}$ into the expressions of Table I, one gets $\bar{P}_{12} / \bar{P}_{11}=0.76$ and $\bar{P}_{22} / \bar{P}_{11}=0.24$. Taking into account the proper value of the direction cosine $\gamma_{1}=0.87$ these intensity ratios become $\bar{P}_{12} / \bar{P}_{11}=0.71$ and $\bar{P}_{22} / \bar{P}_{11}$ $=0.29$ in rather good agreement with the experiment.

The multiple diffraction line observed in the three-beam case $(4 \overline{4} 2,3 \overline{3} 1 ; 1 \overline{1} 1)$ is not frequently found in the Laue patterns of topographs taken from curved crystals. Usually the spots look like those in Fig. 2. There is a number of curved lines crossing each other moving across the spot when the bending in changing. It is thought that these lines except for the vertical one are due to a small unintentional twisting of the crystal. The broad line $A$ is interpreted as a $\left(\overrightarrow{\mathbf{H}}_{2}, \overrightarrow{\mathbf{H}}_{1}, \overrightarrow{\mathbf{H}}_{3}\right)$ triple diffrac-

\begin{tabular}{|c|c|c|}
\hline & Double diffraction & Single diffraction \\
\hline$i h k l$ & $\bar{P}_{i 2}$ & $\bar{P}_{i 1}$ \\
\hline 0000 & $S_{0}\left(1-Q_{1} x_{0}+g Q_{1}^{2} x_{0}^{2}\right)$ & $S_{0}\left(1-Q_{1} x_{0}+g Q_{1}^{2} x_{0}^{2}\right)$ \\
\hline $13 \overline{3} 1$ & $S_{0} Q_{1} x_{0}\left(1-2 g Q_{1} x_{0}\right)$ & $S_{0} Q_{1} x_{0}\left(1-g Q_{1} x_{0}\right)$ \\
\hline $24 \overline{4} 2$ & $S_{0} Q_{1} x_{0}\left(g Q_{1} x_{0}\right)$ & 0 \\
\hline
\end{tabular}
tion line. Here the reciprocal-lattice vectors $\overrightarrow{\mathbf{H}}_{2}$, $\overrightarrow{\mathbf{H}}_{1}$, and $\overrightarrow{\mathrm{H}}_{3}$ correspond to the (153), $(\overline{2} \overline{4} 2)$, and (311) lattice planes. They are related to each other by equations $\overrightarrow{\mathbf{H}}_{2}-\overrightarrow{\mathrm{H}}_{1}=\overrightarrow{\mathrm{H}}_{3}$ and $\overrightarrow{\mathrm{H}}_{1} \cdot \overrightarrow{\mathrm{H}}_{3}=0$. The tri-

TABLE I. The theoretical intensities of the double and single diffraction beams. $Q_{1}=\left|e F_{1} /\left(4 \pi \epsilon_{0} m c^{2} V\right)\right|^{2} \lambda^{3} \cos ^{2} 2 \theta_{B}, S_{0}$ is the incident intensity, $x_{0}$ is the thickness of the crystal, and $g=\int W^{2}(\Delta) d \Delta$, where $W(\Delta)$ is the distribution function of the mosaic block misalignment. 
ple diffraction in this case occurs at wavelengths near $85 \mathrm{pm}$.

The authors wish to thank Dr. W. Graeff and Dr. G. Materlik for fruitful discussions and Professor Dr. C. Kunz, Director of the HASYLAB, for permission to use synchrotron radiation facilities at the storage ring DORIS. The work has been supported by travel grants from the Academy of Finland (T.T.), the Emil Aaltosen säätiö, and the Jenny ja Antti Wihurin rahasto (K.N.).
${ }^{1}$ V. Kelhä, K. Naukkarinen, M. Tilli, T. Tuomi, and J. DiPersio, Technical Research Centre of Finland, General Division Publication 2 (Helsinki, 1976).

${ }^{2} \mathrm{~K}$. Naukkarinen, Stanford Synchrotron Radiation Activity Report 78/10, VIII-81.

3J. D. Stephenson, T. Tuomi, V. Kelhä, and M. Tilli, Phys. Status Solidi, A 53, 271 (1979).

${ }^{4}$ N. Kato and A. R. Lang, Acta Crystallogr. 12, 787 (1959).

${ }^{5}$ M. Sauvage, J. F. Petroff, and P. Skalicky, Phys. Status
Solidi A $\underline{43}, 473$ (1977).

${ }^{6}$ T. Tuomi, M. Tilli, V. Kelhä, and J. D. Stephenson, Phys. Status Solidi A 50, 427 (1978).

${ }^{7}$ R. M. Moon and C. G. Shull, Acta Crystallogr. 17, 805 (1964).

${ }^{8}$ W. H. Zachariasen, Acta Crystallogr. 18, 705 (1965).

${ }^{9}$ M. Renninger, Z. Phys. 106, 141 (1937).

${ }^{10} \mathrm{G}$. Borrmann and W. Hartwig, Z. Kristallogr. 121, 401 (1965).

${ }^{11}$ E. Wagner, Phys. Z. 21, 632 (1920). 

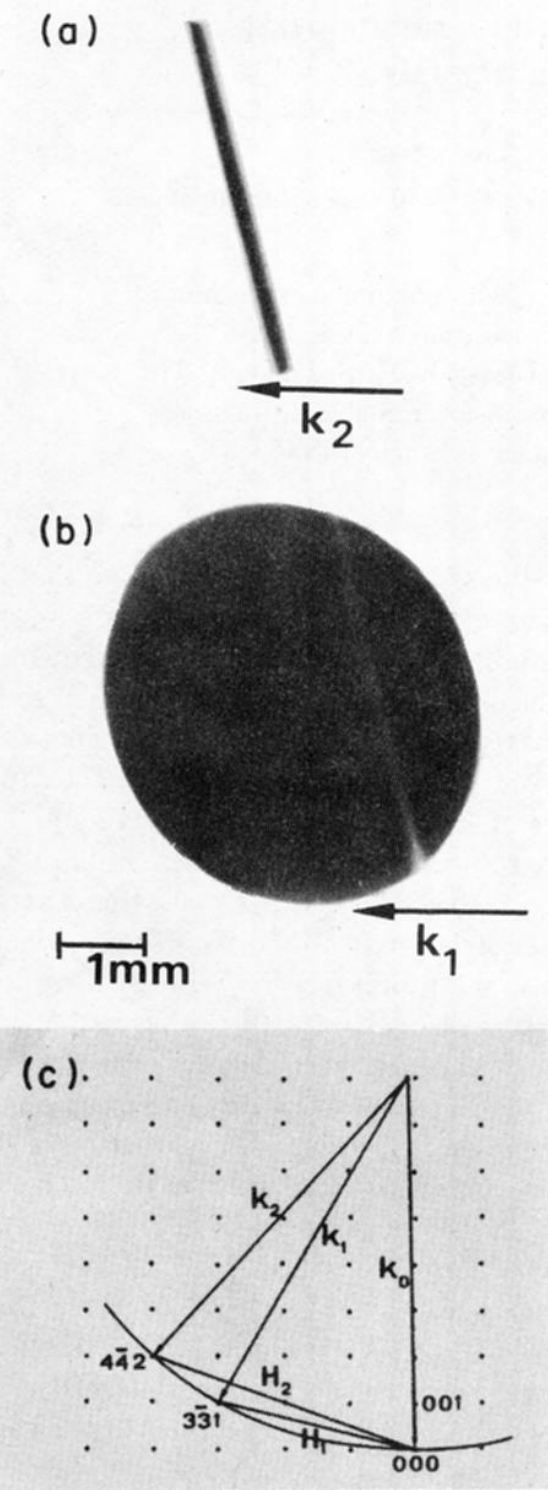

FIG. 1. (a) $4 \overline{4} 2$ and (b) $3 \overline{3} 1$ transmission topographs of a (001) 0.36-mm thick silicon single-crystal plate bent about the vertical [110] axis showing a line at which the double diffraction condition depicted in the reciprocal lattice (c) is fulfilled. The reflection $4 \overline{4} 2$ is forbidden in single diffraction. The exposure time on Kodak $\mathrm{R}$ film was $1 \mathrm{~s}$. The DORIS synchrotron radiation source parameters were $3.3 \mathrm{GeV}$ and $40 \mathrm{~mA}$. The measured strain on the surface of the sample was $3.5 \times 10^{-4}$. 
(a)

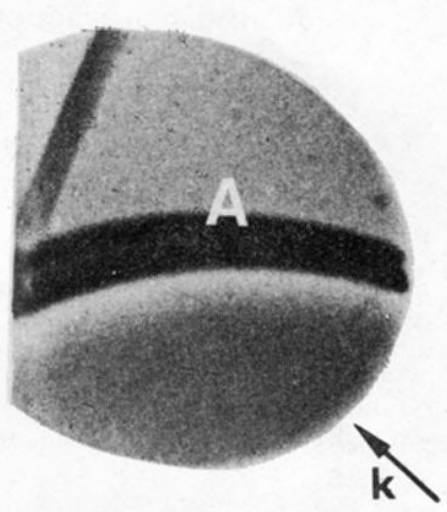

(b)

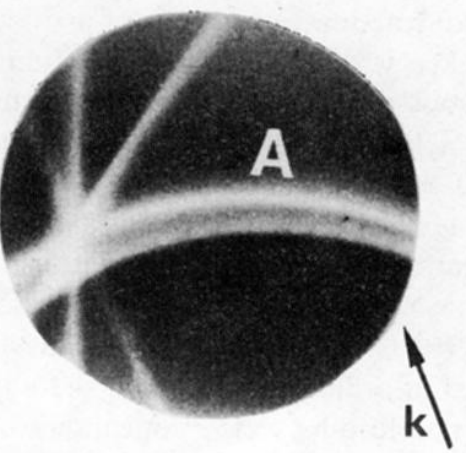

(c)

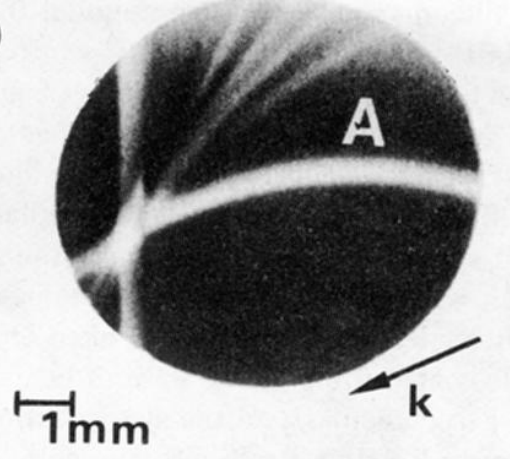

FIG. 2. (a) $1 \overline{5} 3$, (b) $2 \overline{4} 2$, and (c) $3 \overline{1} 1$ transmission topographs of a bent silicon sample showing a triple diffraction line $A$. 